\title{
Juvenile idiopathic arthritis flare due to rice bodies in the knee of a 10-year-old girl
}

\author{
G Faller, ${ }^{1}$ MB BCh, FCPaed (SA), MMed (Paeds); M Haagensen, ${ }^{1}$ MB BCh, FFRad (D) (SA), FRCR; M Barrow, ${ }^{2}$ MB BCh, FCS (Orth) (SA) \\ ${ }^{1}$ Wits Donald Gordon Medical Centre, Johannesburg, South Africa \\ ${ }^{2}$ Sunninghill Hospital, Johannesburg, South Africa

\begin{abstract}
A 10-year-old girl with juvenile idiopathic arthritis in remission presented with a flare of her arthritis. All her joints responded to treatment except the right knee, despite the use of disease-modifying antirheumatic drugs, non-steroidal anti-inflammatory medication and high-dose cortisone. A magnetic resonance imaging scan showed a knee densely packed with rice bodies. After surgical removal of the rice bodies the inflammation settled once again, and the patient remains well on her usual medication.
\end{abstract}

S Afr Med J 2018;108(10):833-835. DOI:10.7196/SAMJ.2018.v108i10.13228

Juvenile idiopathic arthritis (JIA) is arthritis occurring in children aged $<16$ years and characterised by pain, swelling and joint limitation. The International League of Associations for Rheumatology classifies it into six subtypes. ${ }^{[1]}$ Juvenile arthritis is treated with a variety of disease-modifying antirheumatic drugs, intra-articular joint injections and physiotherapy. It has a good prognosis, especially when diagnosed early, and remission is usually achieved, although activity (disease flare) may occur at any time. This article describes a child with JIA who was in remission and off medication when she presented with an unusual flare of disease.

\section{Case report}

A 10-year-old girl presented with increasing joint pain and stiffness in her right knee. She had been known to have JIA (oligoarticular form ${ }^{[1]}$ ) from the age of 3 years, when she had presented with a swollen knee for the first time. She was antinuclear antibody-positive (1:80), and when drainage and intra-articular steroids had not controlled the inflammation she had been treated with methotrexate (MTX). At the time of this presentation, she had been off MTX for the past 18 months and had been in remission for 15 months.

The patient had been seen 3 months previously with relapsed synovitis of the right knee. At that time, she was given an injection of methylprednisolone acetate $40 \mathrm{mg}$ into the affected knee and was put onto leflunomide, as she had experienced side-effects on MXT (nausea and vomiting) and did not want to restart it. She had been taking the leflunomide according to instructions, but was using increasing amounts of non-steroidal anti-inflammatory medication to control the pain. She was unable to take part in her usual sporting activities.

Clinical examination revealed synovitis of the right knee with an effusion and decreased range of movement. There was also mild arthritis of the left wrist and left knee, and the left ankle was significantly swollen. She was otherwise in good health with normal vital signs, a clear chest, normal heart sounds and no hepatosplenomegaly. No rash, nodules or lymphadenopathy were noted. She was ambulatory with a mild antalgic gait. She had been experiencing atopic symptoms and was found to have a post-nasal drip and sinus inflammation by an ENT colleague, who suggested that she restart montelukast sodium and desloratadine, to which she had previously responded for these symptoms.

Blood investigations at this time showed a normal full blood count, an erythrocyte sedimentation rate of $2 \mathrm{~mm} / \mathrm{h}$ and a high
C-reactive protein (CRP) level of $25.3 \mathrm{mg} / \mathrm{L}$ (normal $<5 \mathrm{mg} / \mathrm{L}$ ). In view of the escalation of symptoms and high CRP despite good compliance, the patient was admitted and given an infusion of methylprednisolone sodium succinate $30 \mathrm{mg} / \mathrm{kg}$. She had a partial response with resolution of the synovitis in the wrist, left knee and ankle, but the right knee remained swollen. She was then sent for a magnetic resonance imaging (MRI) scan of the right knee, and at the same time a second infusion of $20 \mathrm{mg} / \mathrm{kg}$ of methylprednisolone sodium succinate was given.

\section{MRI findings}

The MRI study was performed on a 1.5 Tesla Philips Intera MRI scanner. The scan showed a moderate joint effusion with extensive rice-body formation throughout all compartments of the knee joint (Fig. 1). This was evident as small rod-shaped areas of low signal on T1 and T2 sequences. Slightly larger pea-sized loose bodies were noted, mainly in the suprapatellar pouch. The post-contrast sequence also showed markedly enhancing synovium throughout the joint (Fig. 2), but no enhancement of the loose bodies. The articular

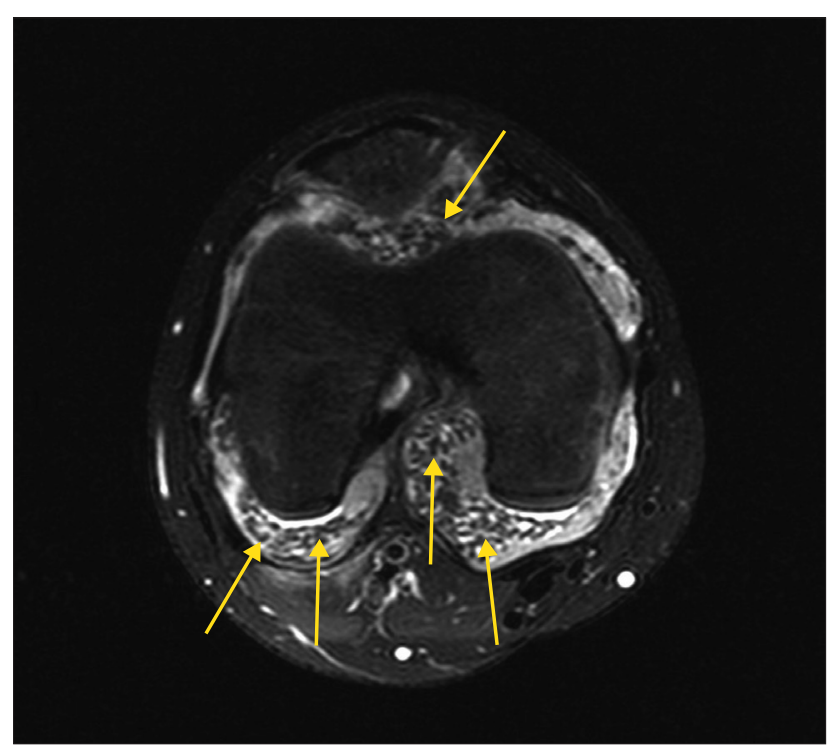

Fig. 1. T2 axial sequence with fat suppression, showing numerous low-signal rice bodies throughout the bright joint effusion (arrows). 
cartilage appeared normal with no erosions or focal marrow oedema. Mildly prominent lymph nodes were noted in the popliteal fossa.

\section{Treatment}

The patient was referred for orthopaedic assessment and a decision was made to remove the rice bodies surgically, as the synovitis and concurrent rice bodies were deemed to be too florid to drain arthroscopically. At surgery (Fig. 3), a $3 \mathrm{~cm}$ medial arthrotomy was performed. Numerous rice bodies were drained, and a synovectomy was performed. The patella and the femoral and tibial hyaline cartilage were soft and there were no erosions. The wound was closed with subcuticular sutures. A surgical drain (Portovac) was placed in the knee and removed after 6 hours. Specimens were sent for histological evaluation to confirm the rice bodies, and for tuberculosis, bacterial and fungal culture. These results confirmed the presence of benign rice bodies with no bacterial or fungal elements and no evidence of tuberculosis. The synovial histology was reported as chronic active synovitis with fibrin deposition probably due to previous haemorrhages into the joint. At follow-up after 2 weeks the patient's knee was less swollen, and her pain had settled.

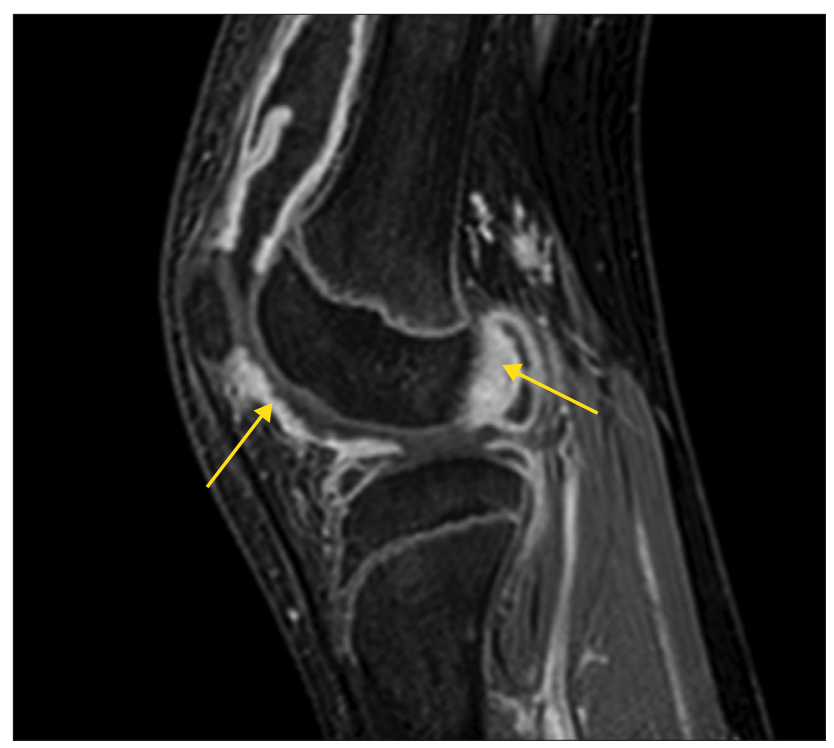

Fig. 2. Sagittal T1 sequence post gadolinium showing marked synovial enhancement (arrows).

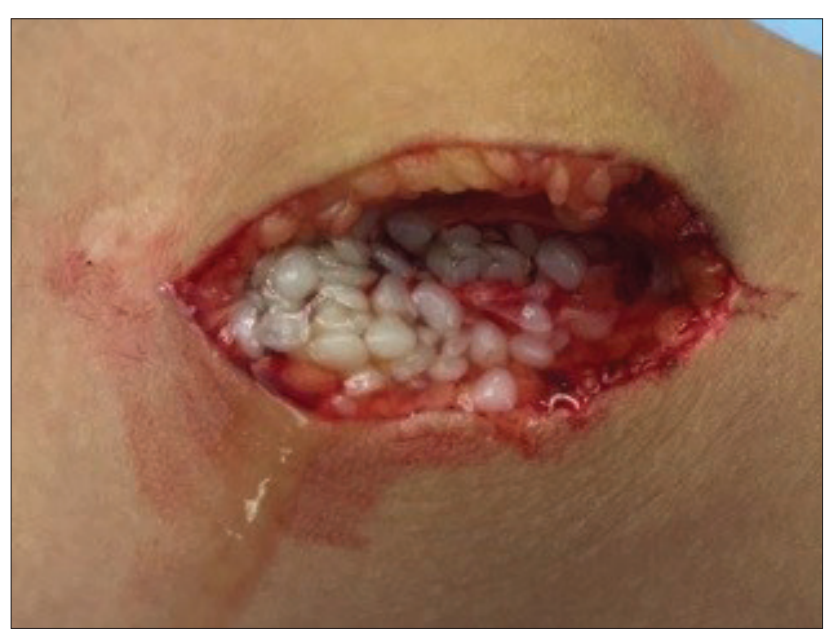

Fig. 3. Rice bodies in the knee at arthroscopy.
Following surgery, the patient's arthritis settled well. She continued on the leflunomide and received physiotherapy and biokinetics assistance for mobility and to rebuild her strength. An MRI scan done 8 months later showed no recurrence of the rice bodies, and she is now in remission, pain free and actively participating in her favourite sports.

\section{Ethical considerations}

The unusual nature of this presentation was explained to the patient and her parents, and full consent and assent were obtained to prepare her data for publication and to use the photographs taken during surgery as well as the MRI scans. Ethics clearance was obtained from the Human Research Ethics Committee of the University of the Witwatersrand (ref. no. M160275).

\section{Discussion}

Rice bodies are inflammatory nodules made up of fibrin, collagen, inflammatory cells and blood vessels. They were so named because they resemble polished grains of rice, as was seen in the picture taken at surgery in this case. The cause of rice bodies remains unclear, but electron microscopy of these bodies taken for a child with JIA show them to contain lipid and glycogen and to resemble synovial lining cells. ${ }^{[2]}$ Historically they have been considered a nonspecific response to inflammation with synovial proliferation, degeneration or infarction in the joint, and have been described in up to $72 \%$ of adult patients with rheumatoid arthritis. They appear to be unrelated to the duration and severity of the arthritis. ${ }^{[3]}$ Rice bodies are rarely described in children, and in the isolated case reports have been a diagnostic or incidental finding. ${ }^{[4-7]}$ One case was severe enough to require surgical intervention ${ }^{[8]}$ only one other case report was found in which the child presented as a relapse with rice bodies, and this child also required synovectomy. ${ }^{[9]}$ Our patient was not a new presentation and had not been experiencing active synovitis, suggesting that the rice bodies developed acutely in response to an acute flare of disease.

A small number of rice bodies in the joint do not require additional treatment but will respond to treatment of the synovial inflammation. In cases where the joint is filled, as in our patient, they will need to be removed. This is usually done openly or arthroscopically but in one case they were small enough to be aspirated through a 14-gauge needle. ${ }^{[10]}$ Following successful removal, we noted that our patient's arthritis was once again amenable to treatment and she remains in remission.

\section{Learning points}

- When relapses of JIA do not respond to therapy, an alternative cause should be sought, especially in a single joint.

- Rice bodies are an uncommon but important finding in resistant joint disease.

- Removal of the rice bodies is necessary for adequate response to treatment.

Acknowledgements. We thank the Clinical Research Office, Wits Donald Gordon Medical Centre, for assisting with obtaining the ethics approval and the preparation of the manuscript.

Author contributions. GF wrote the clinical case report and discussion and did the literature review, MH provided the pictures and MRI description, and $\mathrm{MB}$ wrote the surgical report. All authors reviewed and contributed to the final manuscript preparation.

Funding. None.

Conflicts of interest. None. 
1. Petty RE, Southwood TR, Manners P, et al. International League of Associations for Rheumatology classification of juvenile idiopathic arthritis: second revision, Edmonton, 2001. J Rheumatol 2004;31(2):390-392.

2. Wynne-Roberts CR, Cassidy JT. Juvenile rheumatoid arthritis with rice bodies: Light and electron microscopic studies. Ann Rheum Dis 1979;38(1):8-13. https://doi.org/10.1136/ard.38.1.8

3. Forse CL, Mucha BL, Zabala Santos ML, Ongcapin EH. Rice body formation without rheumatic disease or tuberculosis infection: A case report and literature review. Clin Rheumatol 2012;31(12):1753-1756. https://doi.org/10.1007/s10067-012-2063-8

4. Chung C, Coley BD, Martin LC. Rice bodies in juvenile rheumatoid arthritis. AJR Am J Roentgenol 1998;170(3):698-700. https://doi.org/10.2214/ajr.170.3.9490956

5. DiVito A, Kan JH. Juvenile idiopathic arthritis with rice bodies. Pediatr Radiol 2008;38(11):1283. https://doi.org/10.1007/s00247-008-0978-7

6. Martini G, Tregnaghi A, Bordin T, Visentin MT, Zulian F. Rice bodies imaging in juvenile idiopathic arthritis. J Rheumatol 2003;30(12):2720-2721.
7. Okura Y, Tsumagari S, Nawate M, Yoshioka M, Shikano T, Takahashi Y. Juvenile idiopathic arthritis with rice bodies in a 2-year-old girl. J Pediatr 2016;172:220 https://doi.org/10.1016/j.jpeds.2016.01.039 8. Mutlu H, Silit E, Pekkafali Z, et al. Multiple rice body formation in the subacromial-subdeltoid bursa and knee joint. Skeletal Radiol 2004;33(9):531-533. https://doi.org/10.1007/s00256-004-0757-y

9. Teramoto A, Watanabe K, Kii Y, et al. Recurrent knee arthritis diagnosed as juvenile idiopathic arthritis 9. Teramoto $\mathrm{A}$, Watanabe $\mathrm{K}$, Kii $Y$, et al. Recurrent knee arthritis diagnosed as juvenile idiopathic arthritis
with a 10-year asymptomatic period after arthroscopic synovectomy: A case report. J Med Case Rep with a 10-year asymptomatic period after arthroscopic
2013;7(1):1-5. https://doi.org/10.1186/1752-1947-7-166

10. Cox A, Allen R, Akikusa J. Rice bodies in juvenile idiopathic arthritis: A clinical image. J Paediatr Child Health 2012;48(3):280. https://doi.org/10.1111/j.1440-1754.2012.02420.x

Accepted 23 April 2018. 